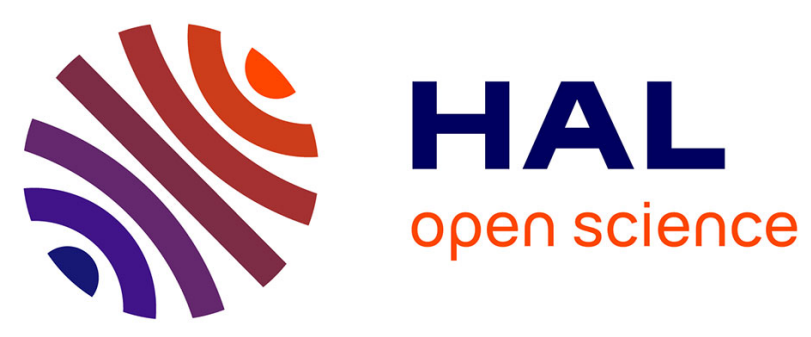

\title{
Multiscale time-resolved fluorescence study of a glycogen phosphorylase inhibitor combined with quantum chemistry calculations
}

\author{
Valentin Maffeis, Konstantinos Mavreas, Filippo Monti, Michael Mamais, \\ Thomas Gustavsson, Evangelia Chrysina, Dimitra Markovitsi, Thanasis \\ Gimisis, Alessandro Venturini
}

\section{To cite this version:}

Valentin Maffeis, Konstantinos Mavreas, Filippo Monti, Michael Mamais, Thomas Gustavsson, et al. Multiscale time-resolved fluorescence study of a glycogen phosphorylase inhibitor combined with quantum chemistry calculations. Physical Chemistry Chemical Physics, 2019, 21 (14), pp.7685-7696. 10.1039/C8CP07538G . cea-02096313

\section{HAL Id: cea-02096313 https://hal-cea.archives-ouvertes.fr/cea-02096313}

Submitted on 23 Oct 2019

HAL is a multi-disciplinary open access archive for the deposit and dissemination of scientific research documents, whether they are published or not. The documents may come from teaching and research institutions in France or abroad, or from public or private research centers.
L'archive ouverte pluridisciplinaire HAL, est destinée au dépôt et à la diffusion de documents scientifiques de niveau recherche, publiés ou non, émanant des établissements d'enseignement et de recherche français ou étrangers, des laboratoires publics ou privés. 
Check for updates

Cite this: Phys. Chem. Chem. Phys., 2019, 21, 7685

Received 10th December 2018 Accepted 1st March 2019

DOI: $10.1039 / c 8 c p 07538 g$

rsc.li/pccp

\section{Multiscale time-resolved fluorescence study of a glycogen phosphorylase inhibitor combined with quantum chemistry calculations $\dagger$}

\author{
Valentin Maffeis, (D) $\ddagger^{a}$ Konstantinos Mavreas, (D) $\ddagger^{b}$ Filippo Monti, (D) \\ Michael Mamais, (D) abd Thomas Gustavsson, (D) a Evangelia D. Chrysina, ${ }^{d}$ \\ Dimitra Markovitsi, (D) $*^{a}$ Thanasis Gimisis $\left(\mathbb{D} *^{b}\right.$ and Alessandro Venturini*c
}

\begin{abstract}
A fluorescence study of $N^{1}$-( $\beta$-D-glucopyranosyl)- $N^{4}$-[2-acridin-9(10H)-onyl]-cytosine (GLAC), the first fluorescent potent inhibitor of glycogen phosphorylase (GP), in neutral aqueous solution, is presented herein. Quantum chemistry (TD-DFT) calculations show the existence of several conformers both in the ground and first excited states. They result from rotations of the acridone and cytosine moieties around an $\mathrm{NH}$ bridge which may lead to the formation of non-emitting charge-transfer states. The fingerprints of various conformers have been detected by time-resolved fluorescence spectroscopy (fluorescence upconversion and time-correlated single photon counting) and identified using as criteria their energy, polarization and relative population resulting from computations. Such an analysis should contribute to the design of new GP inhibitors with better fluorescence properties, suitable for imaging applications.
\end{abstract}

\section{Introduction}

Glycogen phosphorylase (GP), a highly regulated enzyme involved in glycogenolysis, has been used, in recent years, as a molecular target for the development of new antidiabetics. ${ }^{1-6}$ Recently, we reported the synthesis and crystallographic study of a glucose-based acridone derivative, $N^{1}$-( $\beta$-D-glucopyranosyl)$N^{4}$-[2-acridin-9(10H)-onyl]-cytosine (GLAC), which was found to be among the most potent rabbit muscle GP inhibitors. ${ }^{4}$ In addition to its promising therapeutic action, GLAC was shown to be also an interesting optical probe for sensing the enzyme catalytic site. As the lowest in energy electronic absorption band of GLAC is well-separated from those of GP, it provides information about its interaction with the enzyme. Thus, it was shown that, upon binding to GP, GLAC experiences a significantly more basic environment compared to that of the bulk aqueous solvent. ${ }^{4}$

\footnotetext{
${ }^{a}$ LIDYL, CEA, CNRS, Université Paris-Saclay, Gif-sur-Yvette, France. E-mail: dimitra.markovitsi@cea.fr

${ }^{b}$ Department of Chemistry, National and Kapodistrian University of Athens, Athens, Greece.E-mail: gimisis@chem.uoa.gr

${ }^{c}$ Istituto ISOF, Consiglio Nazionale delle Ricerche, Bologna, Italy. E-mail: alessandro.venturini@isof.cnr.it

${ }^{d}$ Institute of Biology, Medicinal Chemistry \& Biotechnology, National Hellenic Research Foundation, Athens, Greece. E-mail: echrysina@eie.gr

$\dagger$ Electronic supplementary information (ESI) available: Synthetic, spectroscopic and theoretical calculation details. See DOI: $10.1039 / \mathrm{c} 8 \mathrm{cp} 07538 \mathrm{~g}$

\# These authors contributed equally.
}

Here, we show that GLAC emits fluorescence both when it is free in neutral aqueous solution and when it is bound to the enzyme. In recent years, the intrinsic fluorescence of inhibitors has been used for the study of their binding to various types of enzymes (see, for example, ref. 7-11). Fluorescence offers another route for sensing the local environment, not only in a static mode, where all effects are averaged, but also in a dynamic way thanks to time-resolved spectroscopy. ${ }^{12-14}$ This has, in particular, been successfully applied to study drug-protein complexes (see, for example, ref. 15-17). To our knowledge, none of the previously reported catalytic site inhibitors of GP provides this possibility.

The most important part of the present work concerns free GLAC in aqueous neutral solution, where it is present in its anionic form, ${ }^{4}$ noted GLAC $^{-1}$ (Fig. 1). It is performed by combining steady-state and time-resolved fluorescence spectroscopies. For the latter, two different detection techniques were used, namely fluorescence upconversion (FU) and time-correlated single photon counting (TCSPC), with the same laser source providing 120 fs pulses at $400 \mathrm{~nm}$. This approach allows us to

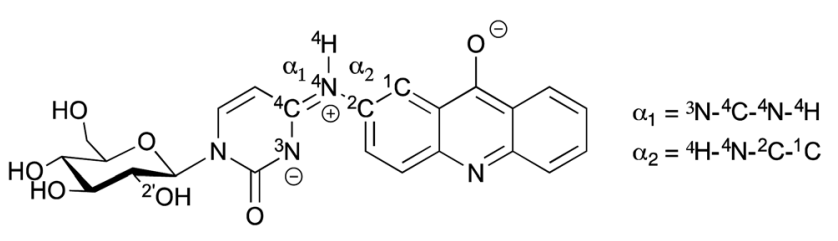

Fig. 1 Schematic representation of $\mathrm{GLAC}^{-1}$ in neutral aqueous solution ( $\alpha_{1}$ and $\alpha_{2}$ designate dihedral angles). 
explore fluorescence decays and fluorescence anisotropies from the femtosecond to the nanosecond time-scales. A few comparative experiments were also performed for acetonitrile solutions, where the neutral form of GLAC is present.

The experimental results are interpreted with the help of quantum-mechanical calculations based on the time-dependent density functional theory (TD-DFT), including implicit solvent via the polarizable continuum model (PCM).

A detailed study of the free GLAC fluorescence is necessary before tackling its behaviour when it is bound to the enzyme. As a matter of fact, its emission properties are strongly affected by conformational factors, related to multiple rotations around the dihedral angles $\alpha_{1}$ and $\alpha_{2}$ (Fig. 1), which give rise to complex potential energy surfaces (PES). Their disentanglement in this first step of the study, will allow us to assess, in a second step, additional factors that intervene in the GLAC/GP complex.

\section{Methodological details}

\subsection{Synthesis}

2-((4-Acetamidophenyl)amino)benzoic acid. To a solution of 2-aminobenzoic acid $(2.77 \mathrm{~g}, 18.6 \mathrm{mmol})$ and 4-aminoacetanilide $(2.79 \mathrm{~g}, 17.7 \mathrm{mmol})$ in $7 \mathrm{ml}$ of diethylene glycol was gradually added, under stirring, anhydrous potassium carbonate $(2.44 \mathrm{~g}$, $17.5 \mathrm{mmol}$ ). After cessation of gas evolution, to the mixture were added freshly precipitated copper $\operatorname{powder}^{18}$ (100 mg, $\left.1.57 \mathrm{mmol}\right)$ and copper(I) oxide (100 mg, $0.70 \mathrm{mmol})$ and the whole mixture was heated under argon at $150{ }^{\circ} \mathrm{C}$ for $8 \mathrm{~h}$. The mixture was cooled to room temperature and filtered. A small portion of sodium metabisulfite was added to the filtrate, which then was acidified with $30 \mathrm{ml}$ of $1 \mathrm{~N} \mathrm{HCl}$. The precipitated solid was filtered, washed with water and dried to yield $2.77 \mathrm{~g}(58 \%)$ of the title compound, as a grayish powder. ${ }^{1} \mathrm{H}$ NMR $\left(200 \mathrm{MHz}, \mathrm{DMSO}-d_{6}\right) \delta 9.94(\mathrm{~s}, 1 \mathrm{H}), 9.53$ $(\mathrm{s}, 1 \mathrm{H}), 7.87$ (dd, $J=7.9,1.6 \mathrm{~Hz}, 1 \mathrm{H}), 7.57$ (d, $J=8.7 \mathrm{~Hz}, 2 \mathrm{H})$, 7.35 (ddd, $J=8.6,7.1,1.7 \mathrm{~Hz}, 1 \mathrm{H}), 7.17$ (d, $J=8.7 \mathrm{~Hz}, 2 \mathrm{H}), 7.08$ (d, $J=$ $8.4 \mathrm{~Hz}, 1 \mathrm{H}), 6.72(t, J=7.5 \mathrm{~Hz}, 1 \mathrm{H}), 2.03(\mathrm{~s}, 3 \mathrm{H})$.

4-Aminoacridone. 2-((4-Acetamidophenyl)amino)benzoic acid (920 mg, $3.40 \mathrm{mmol}$ ) was cyclised and deacetylated by the procedure described in the literature. ${ }^{19}$ The solid which precipitated during neutralisation was filtered, washed with copious amounts of water and dried under vacuum to afford the title compound (580 $\mathrm{mg}, 81 \%$ ) as a greenish-yellow powder. The analytical data were in accordance with those previously reported. ${ }^{4}$

GLAC was prepared following our previously published protocol. ${ }^{4,20}$

\subsection{Spectroscopic measurements}

For the majority of the experiments, a stock solution of GLAC in dimethyl sulfoxide (DMSO, UVASOL Merck, $2.4 \mathrm{mM}$ ) was prepared and aliquots of the stock solution were diluted with ultrapure water (Milli-Q Synthesis) either in a phosphate buffer $\left(0.015 \mathrm{~mol} \mathrm{~L}{ }^{-1} \mathrm{NaH}_{2} \mathrm{PO}_{4}, 0.015 \mathrm{~mol} \mathrm{~L}{ }^{-1} \mathrm{Na}_{2} \mathrm{HPO}_{4}\right.$; pH 7; ingredients from Fluka), or in acetonitrile (UVASOL Merck). For the alkaline solution $(\mathrm{pH}=12.7), 0.113 \mathrm{~mol} \mathrm{~L}^{-1}$
$\mathrm{NaOH} / 0.087 \mathrm{~mol} \mathrm{~L}^{-1} \mathrm{KCl}$ (Fluka) were used. The final concentration of DMSO was $\sim 1 \% \mathrm{v} / \mathrm{v}$, in all solutions.

For steady-state and TCSPC measurements, GLAC was studied both in water and in buffer $(\mathrm{pH}=7.00)$, inside $1 \mathrm{~cm} \times 1 \mathrm{~cm}$ quartz cells, and no difference was detected (Fig. S1, ESI $\dagger$ ). For FU measurements, only water solutions were studied. In the latter case, a rotating QZ cell with an optical path of $1 \mathrm{~mm}$ was used. For all fluorescence measurements, the signal obtained for the neat solvent was negligible compared to the GLAC signal.

Absorption was recorded using a PerkinElmer Lambda 900 instrument. Two different instruments were used for fluorescence spectra: a Shimadzu RF 5310 PC and a SPEX Fluorolog-3 fluorimeter equipped with a double monochromator on the emission side, the spectra obtained with the latter apparatus were corrected for the spectral response of the detection system. For the determination of fluorescence quantum yields $(\phi)$ the absorbance at the excitation wavelength was on the order of 0.01 .

The excitation source for time-resolved experiments was the second harmonic (400 nm, $120 \mathrm{fs}$ ) of a Ti-sapphire laser (Coherent MIRA 900). The repetition rate was $76 \mathrm{MHz}$ for $\mathrm{FU}$ but reduced to $4.75 \mathrm{MHz}$ for TCSPC using a pulse picker (Coherent 9200). The homebuilt FU setup is described in detail elsewhere. ${ }^{21}$ The sample fluorescence was collected with two parabolic mirrors and mixed with the residual $800 \mathrm{~nm}$ fundamental beam in a $0.5 \mathrm{~mm}$ type I BBO crystal. The up-converted signal was spectrally filtered by a double-grating monochromator (SPEX 1680, spectral resolution ca. $5 \mathrm{~nm}$ ) and detected by a photomultiplier (Hamamatsu 1527P) coupled to a photon counter (Stanford SR400). The apparatus function was ca. $330 \mathrm{fs}$ (fwhm). The TCSPC setup uses a Becker \& Hickl GmbH SPC630 card controlled by a home-made LabView program. The fluorescence was spectrally resolved by a small monochromator (HR250, Jobin-Yvon) and then detected by a microchannel plate (R1564 U Hamamatsu). Part of the IR laser pulse was detected by a fast photodiode (ThorLabs) for the synchronisation. The instrumental response function was about 60 ps (fwhm), as determined by the Raman line corresponding to the $\mathrm{O}-\mathrm{H}$ stretching of the water molecule.

In both time-resolved experiments (TCSPC and FU), only the vertically polarised fluorescence was detected. Consequently, the parallel $\left(I_{\text {par }}\right)$ and perpendicular $\left(I_{\text {perp }}\right)$ components of the total fluorescence signals were recorded by varying the polarization, vertical or horizontal, of the exciting beam with a zero-order half-wave plate (in the FU setup) or with a Fresnel rhomb (in the TCSPC setup). As the excitation energies measured after the half-wave plate were identical under parallel and perpendicular conditions, the $G$ factor was set equal to 1 (i.e. no corrections were made). The total fluorescence kinetics $F(t)$ and the fluorescence anisotropies $r(t)$ were constructed according to the equations:

$$
F(t)=I_{\text {par }}(t)+2 G I_{\text {perp }}(t)
$$

and

$$
r(t)=\left(I_{\mathrm{par}}(t)-G I_{\text {perp }}(t)\right) /\left(I_{\mathrm{par}}(t)+2 G I_{\text {perp }}(t)\right) .
$$




\subsection{Theoretical part}

The calculations were performed with DFT and its time dependent extension TD-DFT ${ }^{22}$ with implicit water $(\mathrm{PCM})^{23}$ as implemented in Gaussian $16 .^{24}$ The density functional utilised for the optimisation of the conformers in the ground state and in the first excited state was M062X ${ }^{25}$ at the 6-311g** basis set level. The calculations include the solvent effects (PCM) in the LR (linear response) formalism in the case of absorption. ${ }^{26,27}$ On the other hand, emission has been calculated using the SS (State-Specific) model which is more appropriate for dark states with an oscillator strength of almost zero. This procedure uses the SCRF facility "external-iteration" to carry out a non-equilibrium solvation calculation. It involves the relaxation of the ground state density due to the solvent with respect to the specific excitation under investigation. ${ }^{28,29}$

Absorption and emission spectra were calculated at the PCM/ M062X/6-311+g**//PCM/M062X/6-311g** level. For optimization and single point calculations of the $S_{1}$ excited state, the six energetically lowest excited singlet states were used. The stationary points in the ground and excited states were characterized by frequency calculation. Thermochemical analysis (free energy, $\Delta G$ ) was performed at 298.15 K, starting from the frequency calculations.

The charge transfer character of our systems has been evaluated using Ciofini's $D_{\mathrm{CT}}$ charge transfer indexes ${ }^{30}$ in the framework of the Gaussian 16 software package. The index $\left(D_{\mathrm{CT}}\right)$ gives a measure of the nature of the electronic reorganization occurring upon an electronic excitation.

\section{Experimental results}

The lowest-energy absorption band of GLAC in neutral aqueous solution is characterised by a peak at $405 \mathrm{~nm}$ and a shoulder at around $391 \mathrm{~nm}$. The maximum molar absorption coefficient is $5300 \pm 100 \mathrm{~mol}^{-1} \mathrm{~L} \mathrm{~cm}^{-1}$. Excitation at this band gives rise to two poorly resolved broad fluorescent peaks of almost equal intensity, centred at 455 and $474 \pm 2 \mathrm{~nm}$ (63 and $60 \mathrm{kcal} \mathrm{mol}^{-1}$, respectively). Varying the excitation wavelength between $315 \mathrm{~nm}\left(90.8 \mathrm{kcal} \mathrm{mol}^{-1}\right)$ and $435 \mathrm{~nm}\left(65.7 \mathrm{kcal} \mathrm{mol}^{-1}\right)$ does not induce any change either in the shape or in the intensity of the spectrum, corrected for the absorbed photons (Fig. S2a, ESI $\dagger$ ). In Fig. $2 \mathrm{a}$ are shown the absorption and fluorescence spectra on an energy scale; for the conversion, the emission intensity at wavelength $\lambda$ has been multiplied by $\lambda^{2}$.

The fluorescence quantum yield $\phi$, determined for GLAC in the 5-8 pH range, with excitation at $410 \mathrm{~nm}$, using Coumarin 153 in cyclohexane as a reference $(\phi=0.90),{ }^{31}$ is $0.09 \pm 0.01$. In strongly basic solutions ( $>12.7$ ), where GLAC exists as a bis-anion, ${ }^{4}$ fluorescence vanishes nearly completely, its intensity being lower than $2 \%$ compared to that observed for neutral solutions (Fig. 2a). When GLAC is bound to the enzyme, the intensity of the fluorescence spectrum decreases to about one tenth and its shape changes slightly. We still observe two poorly resolved peaks, but, in contrast to free GLAC, the peak at shorter wavelength is more intense.

Time-resolved fluorescence signals were recorded at 430, 465 and $515 \mathrm{~nm}$ (illustrated in Fig. 2b) over three different time scales: 9 ps, 100 ps and 40 ns.

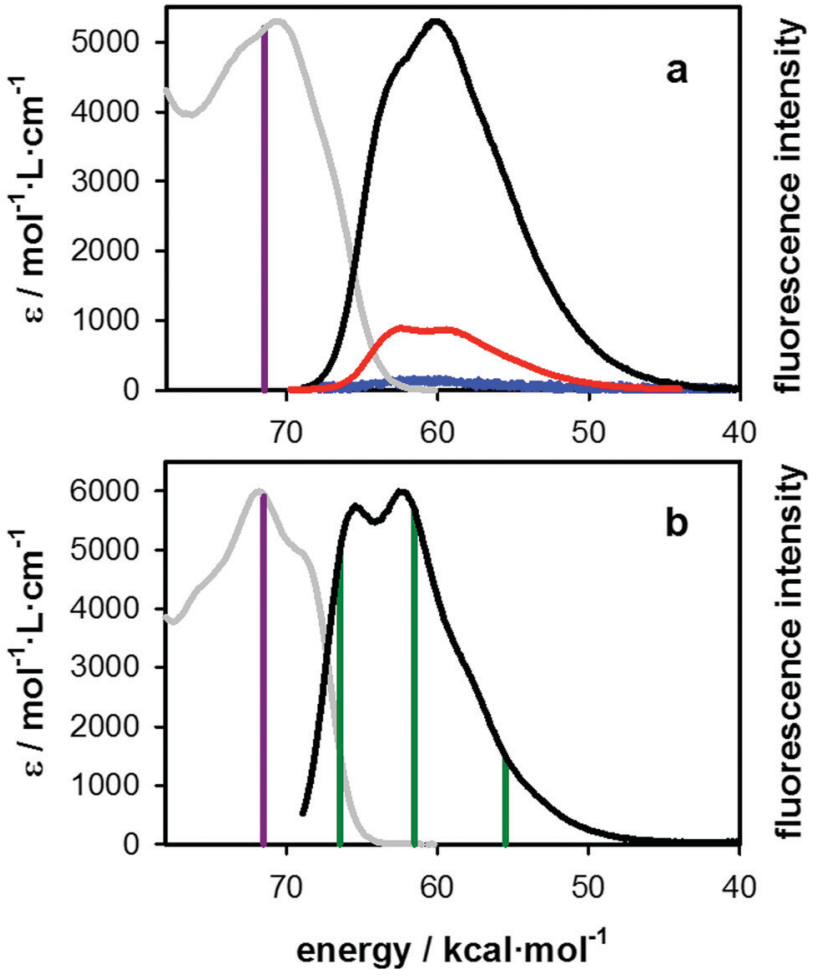

Fig. 2 Steady-state absorption (grey) and corrected fluorescence (black) spectra of GLAC in (a) buffered aqueous solution ( $\mathrm{pH}$ 7) and (b) acetonitrile. The vertical violet lines indicate the excitation wavelength $(400 \mathrm{~nm})$ used in the time-resolved experiments. The red and blue spectra in (a) have been obtained, respectively, for GLAC in $\mathrm{pH} 12.7$ and the GP: GLAC complex in the assay used in ref. 4 (molar ratios $1.0: 0.9$ ). Fluorescence intensities in (a) are representative of the quantum yields. Green vertical lines in (b) correspond to the wavelengths at which time-resolved signals have been recorded. Excitation wavelength: $410 \mathrm{~nm}$.

The total fluorescence intensity exhibits a complex pattern, in particular at early times (Fig. 3a). Within the first $10 \mathrm{ps}$, the signal on the blue side of the emission spectrum loses about $60 \%$ of its amplitude. The opposite behaviour is detected on the red side, where a $10 \%$ rise is observed on the same time-scale. After $20 \mathrm{ps}$, the fluorescence detected at all the probed wavelengths decreases monotonically. On the ns time-scale, the decay at $430 \mathrm{~nm}$ is significantly faster compared to those at 465 and $515 \mathrm{~nm}$, which are rather similar (Fig. 3b). At $40 \mathrm{~ns}$, the signal amplitude is lower than its initial value by more than two orders of magnitude (Fig. S1, ESI $\dagger$ ).

In order to obtain a quantitative description of the fluorescence kinetics, first, the $F(t)$ signals obtained by TCSPC were fitted using tri-exponential model functions, $f(t)=a_{1} \exp \left(-t / \tau_{1}\right)+$ $a_{2} \exp \left(-t / \tau_{2}\right)+a_{0} \exp \left(-t / \tau_{0}\right)$, convoluted by the apparatus function. The parameters derived from the fits are given in Table 1. The values found for the time constant $\tau_{0}$ are well below the time-resolution of the experiment and their weight, $w_{0}=a_{0} \tau_{0} / \sum a_{i} \tau_{i}$, to the TCSCP signals, is negligible $(<0.01)$. As this ultrafast component is resolved by FU, its features are not included in Table 1 . The time constants $\tau_{1}$ and $\tau_{2}$ were found to be the same at all three probed wavelengths, $7.9 \pm 0.1 \mathrm{~ns}$ and 

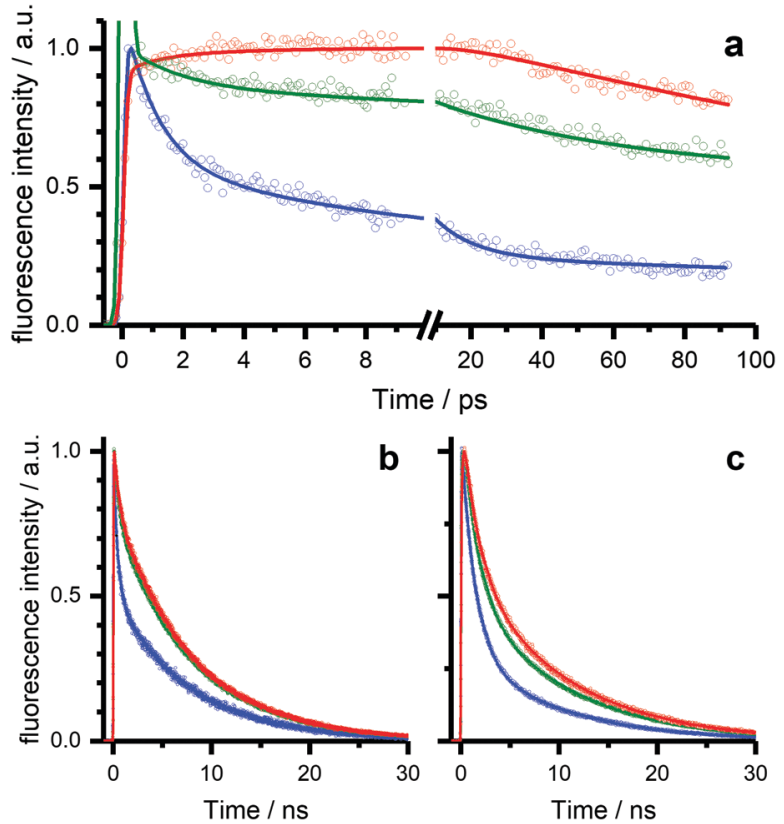

Fig. 3 Total fluorescence of GLAC in neutral aqueous solution (a) and (b) and acetonitrile (c) at 430 (blue), 465 (green) and $515 \mathrm{~nm}$ (red) by fluorescence upconversion (a) and time-correlated single photon counting (b) and (c); circles and solid lines correspond, respectively, to experimental points and fitted functions (see Table 1); excitation wavelength: $400 \mathrm{~nm}$. The green spark in (a) is due to the Raman emission of water.

$0.5 \pm 0.1 \mathrm{~ns}$, respectively, but the corresponding amplitudes are strongly wavelength dependent. It is clear that the fluorescence spectrum is dominated by the longest component, because at its most intense part $w_{1}$ amounts to 0.97 . Even if the amplitude of the shorter component at $430 \mathrm{~nm}$ is 0.5 , its contribution to the total fluorescence at this wavelength is only $6 \%$ and smaller at longer wavelengths.

The $F(t)$ signals obtained by $\mathrm{FU}$ were fitted by fourexponential model function; $f(t)=a_{1} \exp \left(-t / \tau_{1}\right)+a_{2} \exp \left(-t / \tau_{2}\right)+$ $a_{3} \exp \left(-t / \tau_{3}\right)+a_{4} \exp \left(-t / \tau_{4}\right)$, where the two time constants $\tau_{1}$ and $\tau_{2}$ were fixed to the values derived from the TCSPC measurements (Table 1) while $\tau_{3}$ and $\tau_{4}$ were free-floating. The absolute values of the time constants $\tau_{3}(\sim 11 \mathrm{ps})$ and $\tau_{4}(\sim 1 \mathrm{ps})$ are similar for all the probed wavelengths but have opposite signs in the red and the blue part of the spectrum.

The fluorescence anisotropy traces are presented in Fig. 4. All of them decay monotonously, reaching zero in about $1.5 \mathrm{~ns}$.
The initial $r_{0}$ values decrease with increasing emission wavelength. Combined fits of the $r(t)$ signals determined by FU and TCSPC at a given emission wavelength with bi-exponential model functions, $a_{r 1} \exp \left(-t / \tau_{r 1}\right)+a_{r 2} \exp \left(-t / \tau_{r 2}\right)$, were performed.

The resulting fitted parameters are gathered in Table 2, where the values of the time-zero anisotropy $r_{0}$ as well the average depolarization time $\left\langle\tau_{r}\right\rangle=\left(a_{r 1} \tau_{r 1}+a_{r 2} \tau_{r 2}\right) /\left(a_{r 1}+a_{r 2}\right)$ are also shown. The two time constants derived from the fits are similar at the three probed wavelengths: $\tau_{r 1}=280 \pm 10 \mathrm{ps}$ and $\tau_{r 2}=20 \pm 1 \mathrm{ps}$. When going from the blue to the red side of the spectrum, the average depolarization time, determined as $\left\langle\tau_{r}\right\rangle=\left(a_{r 1} \tau_{r 1}+a_{r 2} \tau_{r 2}\right) /\left(a_{r 1}+a_{r 2}\right)$, increases from 121 to $188 \mathrm{ps.}$

Going from neutral aqueous solutions to acetonitrile, both the absorption and the fluorescence spectra shift to higher energies (Fig. 2b), while the fluorescence quantum yield increases from 0.09 to 0.22 . The fluorescence spectra, still independent of the excitation wavelength (Fig. S2b, ESI $\dagger$ ), exhibit now two clearly distinguishable peaks. The fluorescence decays recorded by TCSPC (Fig. 3c) can be fitted by a bi-exponential model function providing two nanosecond components: $\tau_{1}=9.7 \pm 1 \mathrm{~ns}$ and $\tau_{2}=1.3 \pm 1 \mathrm{~ns}$ (Table 1). Both of them are longer than their counterpart in phosphate buffer. This is particularly true for $\tau_{2}$, predominant at the blue part of the spectrum, whose weight increases significantly. Thus, we can reasonably correlate $\tau_{2}$ with the higher energy peak.

\section{Theoretical results}

In order to get a deeper insight into the spectroscopic findings reported above, DFT and TD-DFT calculations have been carried out to map the potential energy surface (PES) of the anionic form of GLAC, both in the ground state $\left(\mathrm{S}_{0}\right)$ and in its lowest electronic excited state $\left(\mathrm{S}_{1}\right)$.

\subsection{Ground state}

In the ground-state, our calculations, identified for $\mathrm{GLAC}^{-1}$ four classes of stable conformers (labelled A, B, C and D) that, for steric hindrance reasons, correspond to seven thermallyaccessible minima labelled as GS-X, where $\mathbf{X}=\mathbf{A}^{\prime}, \mathbf{A}^{\prime \prime}, \mathbf{B}, \mathbf{C}^{\prime}$, $\mathbf{C}^{\prime \prime}, \mathbf{D}^{\prime}$ and $\mathbf{D}^{\prime \prime}$. Moreover, nine transition states were found on $\mathrm{S}_{0}$; they are labelled as TS GS-XY, where $\mathbf{X}$ and $\mathbf{Y}$ are different minima (e.g., TS GS-AB indicates the transition state between GS-A and GS-B).

Table 1 Parameters derived from the fits of total fluorescence signals $F(t)$ obtained by TCSPC and FU with multi-exponential functions $\sum a_{i} \exp \left(-t / \tau_{i}\right)$; $w_{i}=a_{i} \tau_{i} /\left(\sum a_{j} \tau_{j}\right)$ represents the weight of component $i$ to the $F(t)$ determined by each technique

\begin{tabular}{|c|c|c|c|c|}
\hline$\lambda(\mathrm{nm})$ & \multicolumn{2}{|l|}{ TCSPC } & \multicolumn{2}{|l|}{ FU } \\
\hline $430^{a}$ & $7.9 \pm 0.1(0.50,0.94)$ & $0.5 \pm 0.1(0.50,0.06)$ & $11.4 \pm 0.4(0.30)$ & $1.1 \pm 0.1(0.47)$ \\
\hline $515^{a}$ & $7.9 \pm 0.1(0.73,0.97)$ & $0.6 \pm 0.1(0.27,0.03)$ & $-11.5 \pm 1.5(0.11)$ & $-1.1 \pm 0.1(0.07)$ \\
\hline $430^{b}$ & $9.6 \pm 0.1(0.28,0.74)$ & $1.3 \pm 0.1(0.72,0.26)$ & - & \\
\hline $465^{b}$ & $9.7 \pm 0.1(0.44,0.85)$ & $1.3 \pm 0.1(0.56,0.15)$ & - & \\
\hline $515^{b}$ & $9.6 \pm 0.1(0.51,0.88)$ & $1.4 \pm 0.1(0.49,0.12)$ & - & \\
\hline
\end{tabular}

${ }^{a}$ In PBS buffer $(\mathrm{pH}=7) .{ }^{b}$ In acetonitrile. ${ }^{c}$ Large errors due to superposition of the Raman line of water. 


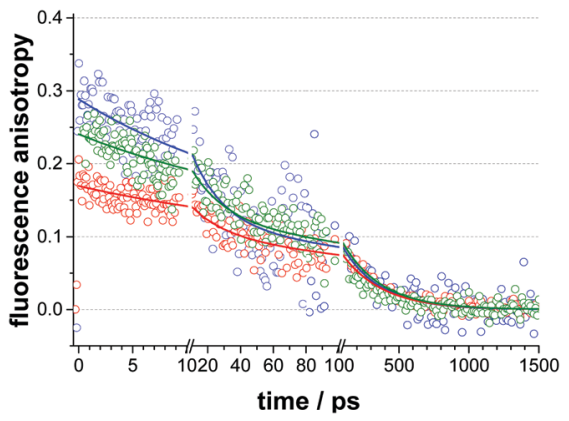

Fig. 4 Fluorescence anisotropy determined for GLAC at 430 (blue), 565 (green) and $515 \mathrm{~nm}$ (red) by fluorescence upconversion and timecorrelated single photon counting; circles and solid lines correspond, respectively, to experimental points and fitted functions (Table 2); excitation wavelength: $400 \mathrm{~nm}$.

Table 2 Parameters derived from combined fits of fluorescence anisotropy $r(t)$ obtained by TCSPC and FU at the same wavelength with biexponential functions $a_{r 1} \exp \left(-t / \tau_{r 1}\right)+a_{r 2} \exp \left(-t / \tau_{r 2}\right)$. Time constants in ps; $\left\langle\tau_{r}\right\rangle=\left(a_{r 1} \tau_{r 1}+a_{r 2} \tau_{r 2}\right) /\left(a_{r 1}+a_{r 2}\right)$ represents the average depolarization time and $r_{0}$ the time-zero anisotropy

\begin{tabular}{lllll}
\hline$\lambda(\mathrm{nm})$ & $\tau_{r 1}\left(a_{r 1}\right)$ & $\tau_{r 2}\left(a_{r 2}\right)$ & $r_{0}$ & $\left\langle\tau_{r}\right\rangle$ \\
\hline 430 & $270 \pm 10(0.12)$ & $19 \pm(0.16)$ & 0.28 & $127 \pm 21$ \\
465 & $280 \pm 10(0.13)$ & $20 \pm(0.11)$ & 0.24 & $161 \pm 20$ \\
515 & $290 \pm 10(0.11)$ & $21 \pm(0.06)$ & 0.17 & $188 \pm 15$
\end{tabular}

The particularly high number of detected conformers is due to all the possible rotations around the two dihedral angles formed by the $\mathrm{NH}$ bridge linking the two planar acridone and cytosine moieties (i.e., $\alpha_{1}$ and $\alpha_{2}$ in Fig. 1). The energy difference between all the conformers is always within $1.5 \mathrm{kcal} \mathrm{mol}^{-1}$, so they are all populated at room temperature. It is worth noting that, in all conformers, the glucose substituent is almost perpendicular to the plane of the cytosine group, to which it is linked, forming a hydrogen bond between the 2 '-OH group of the sugar and the carbonyl oxygen in the cytosine ring.

The asymmetry of the glucose moiety prevents GLAC $^{-1}$ from having a symmetry plane (i.e., the plane of the paper in Fig. 1) even in completely planar conformers (i.e., GS-B). In other cases, for steric hindrance reasons, the molecule is unable to reach complete planarity and couples of almost mirrored minima are found (e.g., GS-A $\mathbf{A}^{\prime}$ and GS-A $\mathbf{A}^{\prime \prime}$ ). In Table S1 (ESI $\dagger$ ) are summarized the data corresponding to all conformers. It can be seen that these pairs of mirrored minima have almost the same geometrical characteristics, energies and spectroscopic properties. In Fig. 5 are depicted all seven ground-state conformers of $\mathrm{GLAC}^{-1}$, grouped as pairs of minima for non-

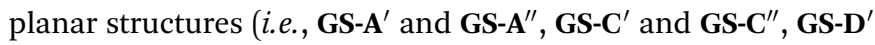
and $\left.\mathbf{G S}-\mathbf{D}^{\prime \prime}\right)$. We also grouped them as geometric $Z-E$ isomers, depending on the orientation around the ${ }^{4} \mathrm{C}-{ }^{4} \mathrm{~N}$ bond $\left(\alpha_{1}\right.$ dihedral $)$, which, in all cases, has a double bond character (see Fig. 1). Ground-state conformers $\mathbf{A}^{\prime}-\mathbf{A}^{\prime \prime}$ and $\mathbf{B}$ are geometric $Z$ isomers, while $\mathbf{C}^{\prime}-\mathbf{C}^{\prime \prime}$ and $\mathbf{D}^{\prime}-\mathbf{D}^{\prime \prime}$ are $E$ isomers.

The rotation around $\alpha_{1}$ requires the system to overcome high energy barriers (e.g., the TS GS-A" $\mathbf{C}^{\prime \prime}$ or TS GS-BD" are located

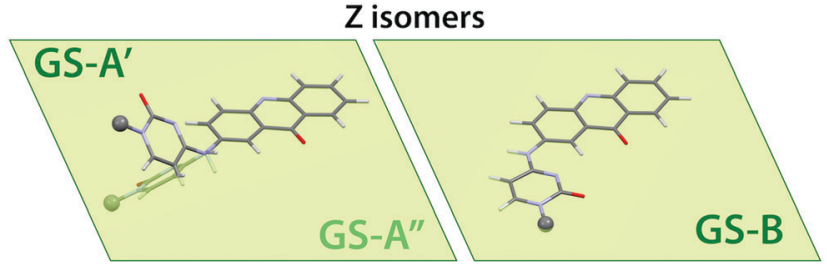

E isomers

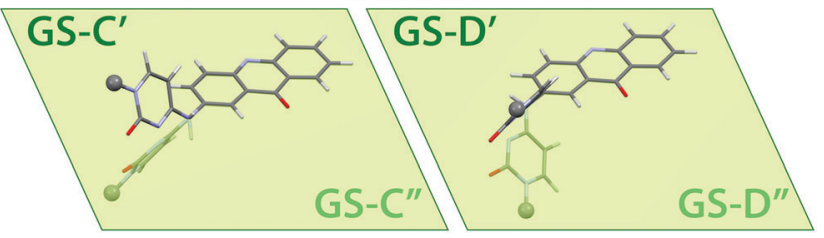

Fig. 5 Ground-state conformer pairs, reflected on a common "acridone plane". The spheres represent the glucose moiety. In all minima, the $\mathrm{NH}$ bridge is virtually coplanar with the cytosine moiety.

around $13 \mathrm{kcal} \mathrm{mol}^{-1}$ above the corresponding minima). This is due to the higher double-bond character of the ${ }^{4} \mathrm{C}-{ }^{4} \mathrm{~N}$ bond, compared to the ${ }^{4} \mathrm{~N}-{ }^{2} \mathrm{C}$ bond. This evidence is corroborated by the shorter average length of the ${ }^{4} \mathrm{C}-{ }^{4} \mathrm{~N}$ bond with respect to the ${ }^{4} \mathrm{~N}-{ }^{2} \mathrm{C}$ one (i.e., $1.35 \AA$ vs. $1.42 \AA$, for all the conformers, see Table S2, ESI $\dagger$ ).

In Fig. 6, the bi-dimensional $S_{0}$ PES (potential-energy surface) of $\mathrm{GLAC}^{-1}$ is reported as a function of $\alpha_{1}$ and $\alpha_{2}$. According to what was stated above, all minima display only two ranges of $\alpha_{1}$ values (indicated with green bands in Fig. 6): one is close

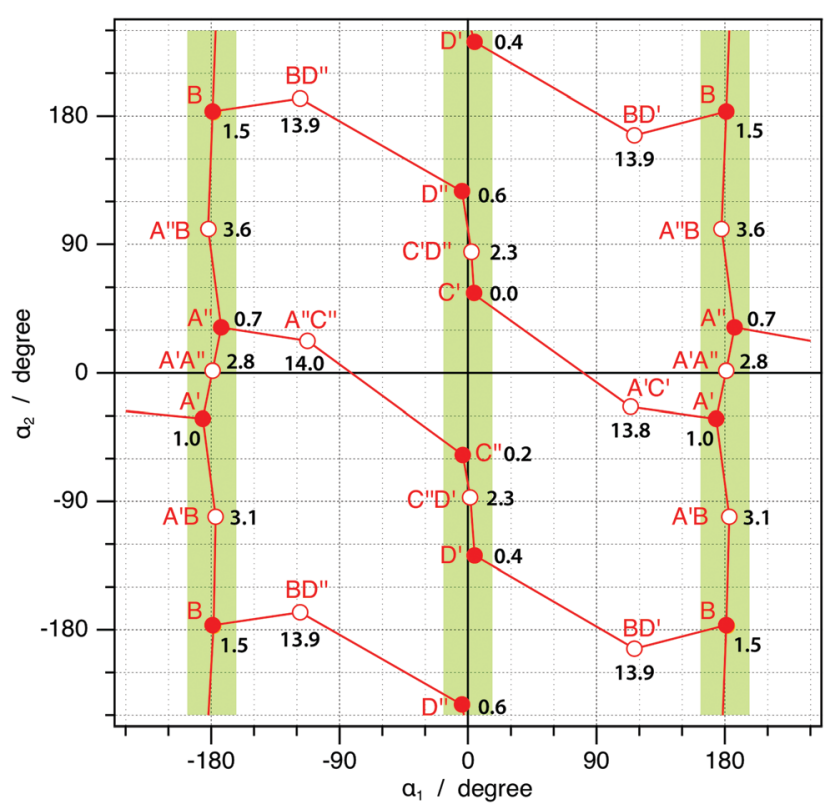

Fig. $6 \mathrm{Bi}$-dimensional conformational PES of $\mathrm{GLAC}^{-1}$ in the ground state. The numbers indicated are the $\Delta \Delta G$ energies in $\mathrm{kcal} \mathrm{mol}^{-1}$ relative to the more stable minimum $\mathbf{C}^{\prime}$. Filled circles are minima and empty circles are transition states. Red lines indicate the real connections between conformers. Also highlighted with a green band, are the zones where $\alpha_{1}$ is almost $0^{\circ}$ or $\pm 180^{\circ}$. In all GS minima, the $\mathrm{NH}$ bridge is virtually coplanar with the cytosine moiety. Notation: $\mathbf{A}^{\prime}$ corresponds to the minimum GS-A' and $\mathbf{A}^{\prime} \mathbf{B}$ to the TS GS-A'B. 
to $0^{\circ}$, corresponding to $E$ conformers (i.e., GS-C ${ }^{\prime}$, GS-C ${ }^{\prime \prime}$, GS-D' and GS- $\mathbf{D}^{\prime \prime}$ ); the other is close to $180^{\circ}$, corresponding to $Z$ conformers (i.e., GS-A', GS-A" and GS-B).

On the contrary, the $\alpha_{2}$ values associated with minima are more spread out and the energy barriers due to the rotation around $\alpha_{2}$ (in which the NH bridge remains coplanar with the cytosine moiety) are always smaller than $4.0 \mathrm{kcal} \mathrm{mol}^{-1}$ (see Fig. 6); this is a strong indication of the single character of the ${ }^{4} \mathrm{~N}-{ }^{2} \mathrm{C}$ bond. It should be emphasised that the previously mentioned mirrored couples of conformers simply differ by an opposite rotation around $\alpha_{2}\left(e . g ., \alpha_{2}=0^{\circ} \pm 32^{\circ}\right.$ in GS-A $\mathbf{A}^{\prime \prime}$ and GS-A', respectively, compare Fig. 5 and 6).

Another important point to stress is that, despite all conformers being virtually reachable from any point of the $\mathrm{S}_{0} \mathrm{PES}$, it is impossible to directly go from GS-C $\mathbf{C}^{\prime}$ to GS-C' ${ }^{\prime \prime}$ and from GS-D' to GS-D' ${ }^{\prime \prime}$. In fact, the two planar transition states TS GS-C' $\mathbf{C}^{\prime \prime}$ $\left(\alpha_{1}=\alpha_{2}=0^{\circ}\right)$ and TS GS-C $\mathbf{C}^{\prime} \mathbf{C}^{\prime \prime}\left(\alpha_{1}=0^{\circ}\right.$ and $\left.\alpha_{2}=180^{\circ}\right)$ were not found, due to the steric hindrance between the hydrogen atoms of the acridone and cytosine moieties.

\subsection{Excited state}

In order to rationalize the excited-state processes experimentally observed in $\mathrm{GLAC}^{-1}$, we investigated the PES of its lowest excited state (i.e., $\mathrm{S}_{1}$ ), starting from the geometries of all seven minima previously found in the ground state. In Fig. 7, the $S_{1}$ PES is depicted as a function of both $\alpha_{1}$ and $\alpha_{2}$ dihedral angles. Upon relaxation from the Frank-Condon regions, we were able to locate ten minima on the $\mathrm{S}_{1}$ PES (see Table S3, ESI $\dagger$ ).

Upon $\mathrm{S}_{0} \rightarrow \mathrm{S}_{1}$ excitation, both GS-A' ${ }^{\prime}$ and GS-A" conformers relax to the same excited-state minimum (i.e., ES-A), which is planar, like the one directly reached from GS-B (i.e., ES-B). These two planar excited-state conformers display the same $\alpha_{1}$ dihedral angle (i.e., $\alpha_{1}=180^{\circ}$ ), but different values of $\alpha_{2}$ (i.e., $\alpha_{2}=0^{\circ}$ or $180^{\circ}$ for ES-A and ES-B, respectively).

On the other hand, the excited-state planarization of $\alpha_{2}$ is not observed upon relaxation of the remaining conformers (i.e., $\mathbf{C}^{\prime}, \mathbf{C}^{\prime \prime}, \mathbf{D}^{\prime}$ and $\mathbf{D}^{\prime \prime}$ ); accordingly, four corresponding minima are also found on the $S_{1}$ potential-energy surface.

It is worth noting that two extra couples of minima (i.e., ES- $\mathbf{E}^{\prime}$ and ES-E $\mathbf{E}^{\prime \prime}$, ES-F $\mathbf{F}^{\prime}$ and $\mathbf{E S}-\mathbf{F}^{\prime \prime}$ ), which do not have their counterparts on $S_{0}$, were found on the $S_{1}$ PES. All the geometries of these new excited-state minima display $\alpha_{1}$ angles around $\pm 90^{\circ}$ (yellow bands in Fig. 7), meaning that the two aromatic moieties of GLAC $^{-1}$ turn out to be mutually orthogonal (Fig. 8), leading to the formation of a twisted intramolecular charge-transfer (TICT) state. These four twisted minima display $\alpha_{2} \approx 0^{\circ}$ or $180^{\circ}$, meaning that the lone pair of the $\mathrm{NH}$ bridge always overlaps with the $\pi$ orbitals of the acridone moiety (see Fig. 8).

Analysis of the $\mathrm{S}_{1}$ PES and of other six states included in the calculation shows that the formation of the four charge-transfer dark states (TICT) does not correspond to crossing of different states but is simply due to a charge-shift between the acridone and cytosine moieties in $\mathrm{S}_{1}$. This charge-shift is also indicated by the calculated $D_{\mathrm{CT}}$ indexes. They show the difference of the non-emitting states with the other $\mathrm{S}_{1}$ minima (see Table $\mathrm{S} 4$ for the values and Fig. S3, ESI $\dagger$ for a graphical representation of the $D_{\mathrm{CT}}$ ).

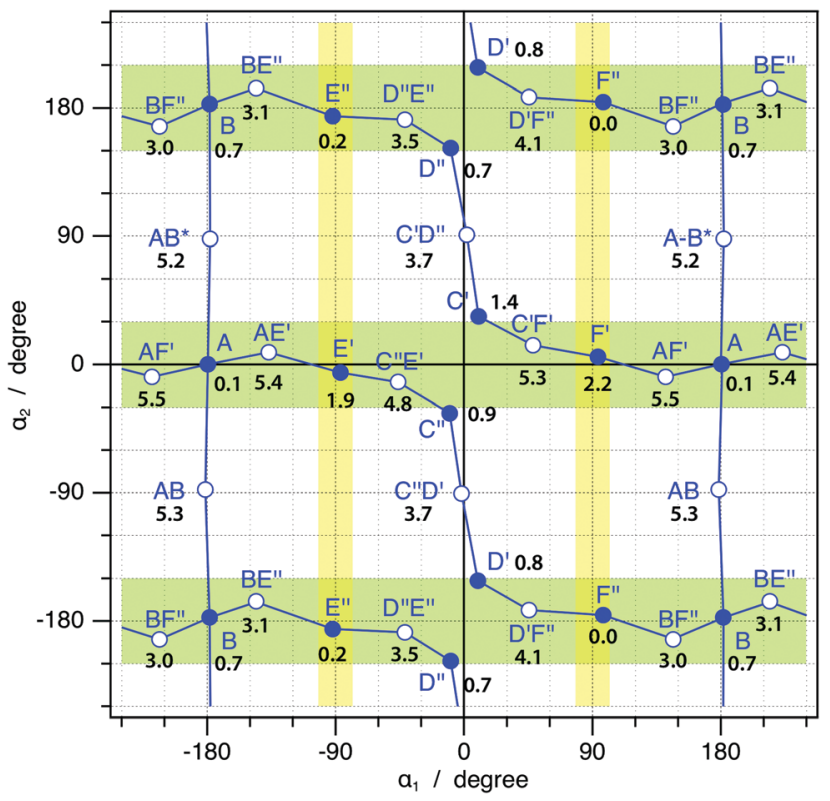

Fig. $7 \mathrm{Bi}$-dimensional PES of $\mathrm{GLAC}^{-1}$ in its lowest excited state (i.e., $\mathrm{S}_{1}$ ). The numbers indicated are the $\Delta \Delta G$ energies in $\mathrm{kcal} \mathrm{mol}^{-1}$ relative to the more stable minimum $\mathbf{F}^{\prime \prime}$. Filled circles are minima and empty circles are transition states. Blue lines indicate the real connections between conformers. We have also highlighted with a green band the zones where $\alpha_{2}$ is almost $0^{\circ}$ or $\pm 180^{\circ}$. In all ES minima, the $\mathrm{NH}$ bridge is close to coplanar with the acridone moiety. Yellow bands indicate the region of the non-emitting species. Notation: A corresponds to the minimum ES-A and $A E^{\prime}$ to the TS ES-AE'.

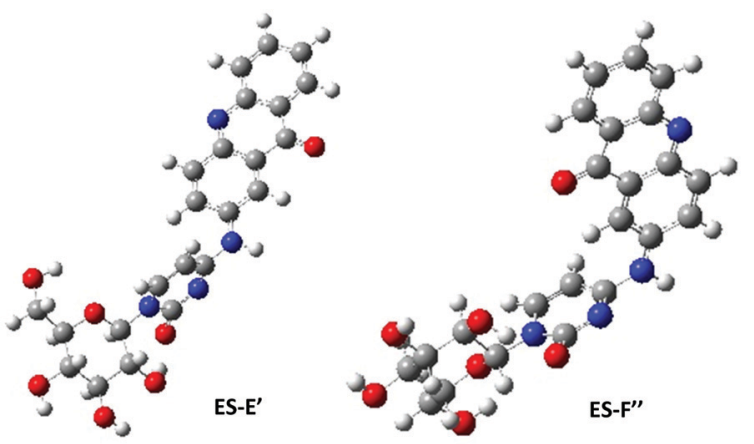

Fig. 8 Fully-optimized minimum-energy geometries of the ES-E' and ES- $F^{\prime \prime}$ conformers on the $S_{1}$ excited-state PES. In both these conformers, the acridone and the cytosine are mutually orthogonal, leading to the formation of a TICT state.

As observed, the $D_{\mathrm{CT}}$ indexes of the non-emitting species show a spatial charge separation significantly higher than for the other minima, indicating the above-mentioned charge-shift.

All the $\mathrm{S}_{1}$ conformers have similar energies and their difference is not higher than $2.2 \mathrm{kcal} \mathrm{mol}^{-1}$, with the twisted conformer ES-F" $\mathbf{F}^{\prime \prime}$ being the most stable one (see Table S3, ESI $\dagger$ ). In comparison with the ground state PES, the rotational barriers around $\alpha_{1}$ decrease and those around $\alpha_{2}$ increase slightly. Moreover, both the average distances of the ${ }^{4} \mathrm{~N}^{2} \mathrm{C}$ and ${ }^{4} \mathrm{~N}-{ }^{4} \mathrm{C}$ bonds are $1.38 \AA$, for all $\mathrm{S}_{1}$ conformers. This indicates that, in the excited state, both the ${ }^{4} \mathrm{~N}-{ }^{2} \mathrm{C}$ and ${ }^{4} \mathrm{~N}-{ }^{4} \mathrm{C}$ bonds are even longer than the 
typical partial double amidic bond (i.e., approx. $1.32 \AA$ ), suggesting that free rotation around both $\alpha_{1}$ and $\alpha_{2}$ is allowed and that the formation of stable twisted minima becomes favoured (see Table S2, ESI $\dagger$ ).

\subsection{Spectroscopic properties}

Electronic absorption. For each of the seven ground-state conformers, we calculated, through TD-DFT methods, the six lowest-energy electronic transitions. For the sake of clarity, only the properties of the $S_{0} \rightarrow S_{1}$ electronic transition are reported in Table 3 for all conformers.

For all the ground-state conformers, the $\mathrm{S}_{0} \rightarrow \mathrm{S}_{1}$ electronic transition can be described as an almost pure HOMO $\rightarrow$ LUMO excitation. Since the HOMO is mainly localized on the acridone and the LUMO is delocalized over both the acridone and the cytosine moieties (Fig. 9), the $\mathrm{S}_{0} \rightarrow \mathrm{S}_{1}$ transition has a mixed character. On the one hand, there is a $\pi-\pi^{*}$ local-excited (LE) contribution from the acridone $\pi$ system; on the other hand, a charge-transfer (CT) component implies the excitation of one electron from the acridone to the cytosine moiety.

For values of the dihedral angle $\alpha_{2}$ far from zero, like for conformers GS-D' ${ }^{\prime}$ and GS-D ${ }^{\prime \prime}\left(\alpha_{2}=128.0^{\circ}\right.$ and $127.5^{\circ}$, Table 3) the LE character prevails since both $\pi-\pi^{*}$ orbitals are localized on the acridone.

The $\mathrm{S}_{0} \rightarrow \mathrm{S}_{1}$ transition energy, computed at the M062X level, is approx. $75.4 \mathrm{kcal} \mathrm{mol}^{-1}$ for all conformers in water and only a minor red shift is observed in the case of the planar conformer

Table 3 Relevant structural and spectroscopic parameters computed for all GLAC ${ }^{-1}$ ground-state conformers: dihedral angles $\alpha_{1}$ and $\alpha_{2}$; Boltzmann population of the different ground-state conformers at $300 \mathrm{~K}$; energy $(E)$ and oscillator strength $(f)$ corresponding to the $S_{0} \rightarrow S_{1}$ electronic transition; and population in the Franck-Condon state, determined as $p_{\mathrm{FC}}=100 \cdot p_{\mathrm{GS} i} \cdot f_{i} / \sum p_{\mathrm{GS} i} \cdot f_{i}$

\begin{tabular}{lrrrlll}
\hline Isomer & Angle $\alpha_{1}$ & Angle $\alpha_{2}$ & $p_{\mathrm{GS}}(\%)$ & $E\left(\mathrm{kcal} \mathrm{mol}^{-1}\right)$ & $f$ & $p_{\mathrm{FC}}(\%)$ \\
\hline GS-A $^{\prime}$ & +173.9 & -32.1 & 6.0 & 75.4 & 0.162 & 8 \\
GS-A $^{\prime \prime}$ & -173.2 & +32.1 & 9.9 & 75.3 & 0.164 & 13 \\
GS-B $^{-179.1}$ & +176.7 & 2.5 & 74.0 & 0.085 & 2 \\
GS-C $^{\prime}$ & +4.2 & +56.2 & 31.5 & 75.7 & 0.133 & 33 \\
GS-C $^{\prime \prime}$ & -3.8 & -57.4 & 22.3 & 75.7 & 0.131 & 23 \\
GS-D $^{\prime}$ & +4.5 & -128.0 & 16.3 & 75.5 & 0.099 & 13 \\
GS-D $^{\prime \prime}$ & -4.3 & +127.5 & 11.5 & 75.5 & 0.100 & 9
\end{tabular}

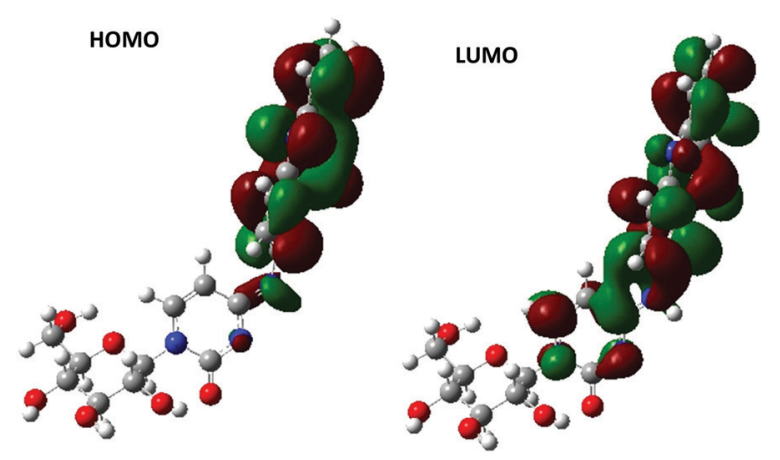

Fig. $9 \mathrm{HOMO}$ and LUMO orbitals, corresponding to the lowest transition of the most stable conformer GS-C'.
Table 4 Emission properties computed at the M062X level for the $\mathrm{GLAC}^{-1}$ conformers in water (using PCM); emission energy $(E)$, oscillator strength $(f)$ and Stokes shift (kcal $\left.\mathrm{mol}^{-1}\right)$

\begin{tabular}{llll}
\hline Conformer & $E\left(\mathrm{kcal} \mathrm{mol}^{-1}\right)$ & $f$ & Stokes shift \\
\hline ES-A & 67.4 & 0.153 & 7.9 \\
ES-B & 69.3 & 0.088 & 4.7 \\
ES-C $^{\prime}$ & 69.0 & 0.114 & 6.7 \\
ES-C $^{\prime \prime}$ & 69.1 & 0.118 & 6.6 \\
ES-D $^{\prime}$ & 68.9 & 0.087 & 6.6 \\
ES-D & 68.8 & 0.096 & 6.7 \\
ES-E & 5.6 & 0.000 & \\
ES-E & 9.1 & 0.001 & \\
ES-F & 9.2 & 0.001 & \\
ES-F $^{\prime \prime}$ & 8.7 & 0.001 & \\
\end{tabular}

GS-B (i.e., $74.0 \mathrm{kcal} \mathrm{mol}^{-1}$, see Table 3). This conformer is characterised by $f=0.085$, the lowest oscillator strength; $f$ increases for the other mirrored couples of conformers, following the trend GS-A > GS-C > GS-D.

Emission. Apart from the two ES-E and ES-F couples, the relaxation from the Franck-Condon region to the excited state minimum involves a charge shift from acridone to cytosine. The two linear GS-A conformers emit from the same ES-A planar conformer, at $65.7 \mathrm{kcal} \mathrm{mol}^{-1}$ with an oscillator strength of 0.199 (Table 4).

It is certain, although in a qualitative manner, that the transformation of the two ground state minima in the excited state surface is coupled with the second vibrational mode $\alpha_{2}$ of the two GS-A minima (e.g., $15.85 \mathrm{~cm}^{-1}$ for GS-A'). This normal mode is due to the opposite rotation of the two aromatic groups around the $\mathrm{NH}$ bridge. GS-B remains planar in the excited state and the normal vibrational mode corresponding to the rotation between the two aromatic groups is the first at $14.01 \mathrm{~cm}^{-1}$.

This behaviour is similar in the two couples GS-C and GS-D, where this transformation is coupled with the first vibrational mode (e.g., $\nu_{1}$ at $10.46 \mathrm{~cm}^{-1}$ for GS-C' $\mathbf{C}^{\prime \prime}$ and $\nu_{1}$ at $14.83 \mathrm{~cm}^{-1}$ for GS-D', respectively, see Fig. 10) and is due to rotation around the $\alpha_{2}$ angle.

The steric hindrance between the hydrogens of the acridone moiety $\left(\mathrm{C}-\mathrm{1}^{\prime \prime}\right.$ or $\left.\mathrm{C}-3^{\prime \prime}\right)$ and the hydrogen of the $\mathrm{NH}$ bridge, on the one hand hinders planarisation of the structure, and on the other favours transition toward the non-emitting couples of minima ES-E and ES-F (emission at around 6-9 $\mathrm{kcal} \mathrm{mol}^{-1}$ with an oscillator strength of 0.00 ). The two aromatic planes rotate in opposite directions becoming orthogonal. The orthogonality of the two planes results in a lack of any overlap between the

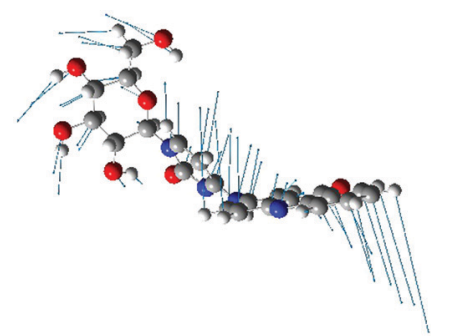

Fig. 10 Lower vibrational mode computed for the GS-C" minimum. 

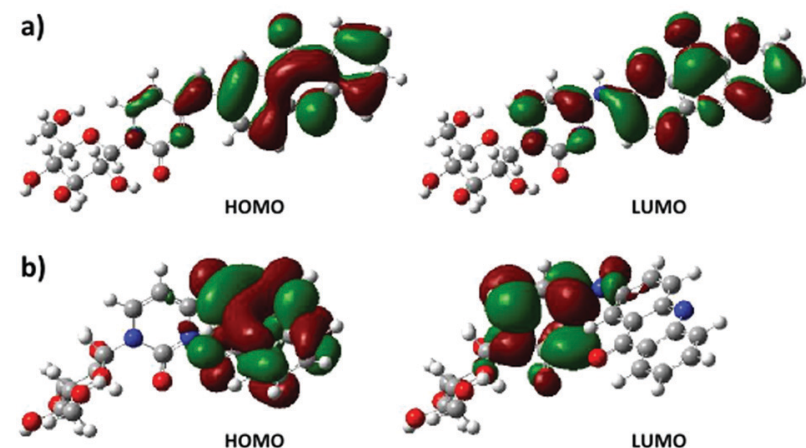

Fig. 11 HOMO and LUMO orbitals: (a) in the planar excited-state conformer ES-A, the orbitals are delocalized over the two aromatic rings. (b) In the twisted non-emissive conformer ES-F, the HOMO is localized on the acridone while the LUMO is on the cytosine moiety.

HOMO and LUMO, corresponding to a charge transfer transition from acridone to the cytosine moiety. The difference between the orbitals of one emitting species (ES-D') and one non-emitting species $\left(\mathbf{E S}-\mathbf{F}^{\prime \prime}\right)$ is illustrated in Fig. 11. The excited state surface shows the existence of non-emitting species next to various emitting conformational minima. The nature of the dark states, evaluated using the SS (State-Specific) model, suggests the presence of conical intersections close to the $\mathrm{S}_{1}$ minima. In fact, the evaluated emission energies are about $7 \pm 2 \mathrm{kcal} \mathrm{mol}^{-1}$, which are in the IR region where a conical intersection can be reached, thanks to vibrational coupling between $S_{0}$ and $S_{1}$.

In general, TD-DFT methods cannot provide an accurate description of the conical intersections and the investigation of the deactivation dynamics, possibly requiring wave-function multiconfigurational methods, is out of the scope of the present work.

The excited state description of the $\mathrm{S}_{1}$ surface has been validated using a long-range density functional like CAM-B3LYP. ${ }^{32}$ The studied systems, ES-A, TS ES-AF ${ }^{\prime}$ and ES-F' ${ }^{\prime}$, show the same characteristics (geometries, orbitals and transition energies) as those derived using the M062X functional (see Table 4 and Fig. S4, ESI $†$ ).

In Fig. 12 are gathered both the ground-state and the lowest excited-state potential energy surfaces of $\mathrm{GLAC}^{-1}$, reported as a function of both $\alpha_{1}$ and $\alpha_{2}$ dihedral angles. This overlap between the $S_{0}$ and $S_{1}$ PESs allows an easier evaluation of all the possible deactivation pathways that the system may follow right after vertical excitation from $S_{0}$ minima to $S_{1}$.

As an example, the position of GS-D' $\mathbf{D}^{\prime}$ in the ground state corresponds to its Frank-Condon position after absorption and makes it possible to see which minima of the excited state can be reached easily. In this case, the more easily accessible minimum is ES-D ${ }^{\prime}$ which can evolve to ES-C ${ }^{\prime \prime}$ or to the most stable minimum of the $\mathrm{S}_{1}$ surface, the non-emitting species ES-F', through an energy barrier of $3.3 \mathrm{kcal} \mathrm{mol}^{-1}$.

The PESs of the ground and excited states provide a detailed picture of the photophysical behaviour of the $\mathrm{GLAC}^{-1}$ in water. The flat PES of the excited state, where emitting and non-emitting species are directly connected, is due to a reorganisation involving mutual rotation around the two dihedral angles $\alpha_{1}$ and $\alpha_{2}$ which provokes, alternatively, localisation and delocalisation of the $\pi$ HOMO and LUMO orbitals involved in the excitation.

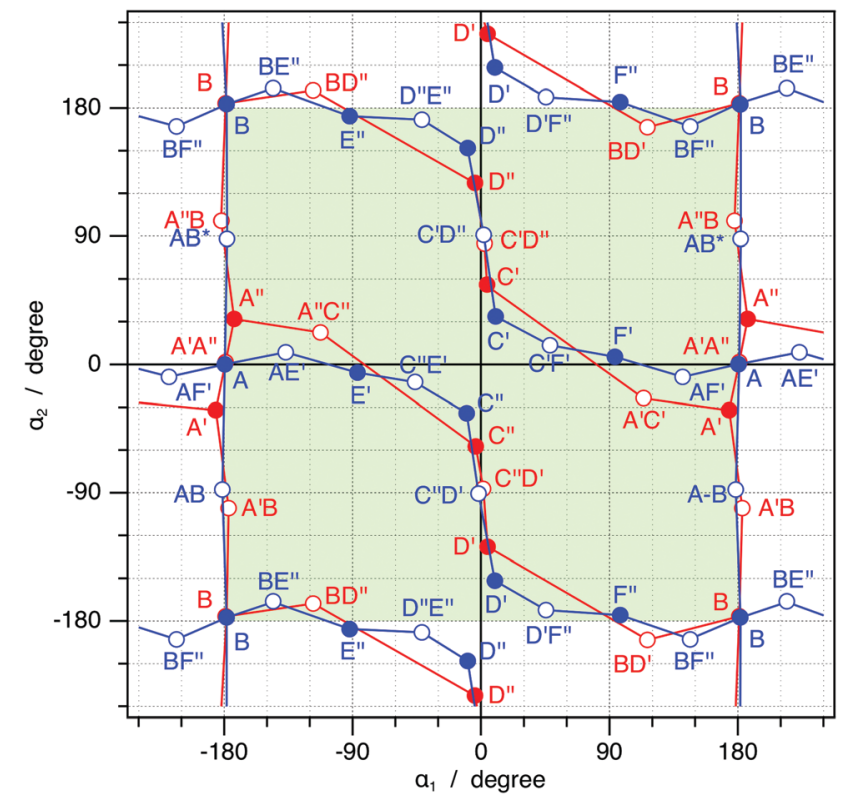

Fig. 12 PES of the ground state $S_{0}$ and of the first excited state $S_{1}$ determined for the various conformers of $\mathrm{GLAC}^{-1}$. Filled circles are minima and empty circles are transition states with the corresponding lines describing the real connections between them (red: ground state; blue: excited state). The picture is an overlap of Fig. 6 and 7.

Table 5 Fluorescence anisotropies computed at the DFT M062X level for various combinations of absorbing and emitting conformers of $\mathrm{GLAC}^{-1}$

\begin{tabular}{lcrrr}
\hline & $A_{\text {abs }}$ & \multicolumn{1}{c}{$B_{\text {abs }}$} & \multicolumn{1}{c}{$C_{\text {abs }}$} & \multicolumn{1}{c}{$D_{\text {abs }}$} \\
\hline$A_{\text {emi }}$ & 0.21 & 0.12 & -0.19 & -0.15 \\
$B_{\text {emi }}$ & -0.02 & 0.40 & -0.16 & -0.18 \\
$C_{\text {emi }}$ & -0.19 & -0.16 & 0.35 & -0.19 \\
$D_{\text {emi }}$ & -0.19 & 0.11 & -0.18 & 0.17 \\
$E_{\text {emi }}$ & -0.16 & -0.20 & 0.20 & -0.20 \\
$F_{\text {emi }}$ & -0.12 & -0.11 & 0.07 & 0.23 \\
\hline
\end{tabular}

This structural rearrangement corresponds to a decrease of the ${ }^{4} \mathrm{~N}-{ }^{4} \mathrm{C}$ bond order coinciding with an increase of the ${ }^{4} \mathrm{~N}-{ }^{2} \mathrm{C}$ bond order. In the ground state, rotation around $\alpha_{1}$ has a quite large barrier which inhibits mixing between $Z$ and $E$ isomers. In the $S_{1}$ surface these barriers are lower by at least one third and in principle could permit the photoinduced isomerization of the molecule. Moreover, the analysis of the excited state orbitals shows that rotation around the two aromatic planes defined by the acridone and cytosine moieties, leads to the formation of dark states; this is due to non-overlapping HOMO-LUMO orbitals and happens when the dihedral angle $\alpha_{2}$ is close to $90^{\circ}$ (Fig. 9).

Finally, the fluorescence anisotropy was calculated using the formula $r_{0}=2\left(\left(3 \cos ^{2} \beta-1\right) / 2\right) / 5$, where $\beta$ is the angle between the absorption and emission dipoles (Table 5).

\section{Discussion}

The experimental part of our work showed that $\mathrm{GLAC}^{-1}$ in neutral aqueous solution emits fluorescence with a quantum 
yield of 0.09 . This is about ten times lower than that of acridone (ca. 1$)^{33}$ but much higher than that of cytosine $\left(c a \cdot 10^{-4}\right),{ }^{34}$ suggesting the existence of important electronic interactions between the two aromatic moieties of the compound. Our computational part rationalised these interactions and revealed a very rich conformational landscape, both in the ground state and in the first excited state. The properties of the computed potential energy surfaces will help us to explain the complex behaviour observed by our time-resolved measurements.

Our calculations identified four classes of stable conformers (i.e., a planar conformer and three couples of mirrored ones) that we called GS-A, GS-B, GS-C and GS-D, differing in the way that the two aromatic moieties are rotated with respect to each other (Fig. 5). The ground state populations at room temperature, $p_{\mathrm{GS}}$, were found to be $15.9,2.5,53.8$ and $27.8 \%$, respectively, for GS-A, GS-B, GS-C and GS-D. Isomerisation is governed by two independent rotations around the angles $\alpha_{1}$ and $\alpha_{2}$ and may lead to the formation of non-emitting TICT states, for which the two planar groups are mutually orthogonal. We identified two couples of twisted intramolecular charge transfer (TICT) states (ES-E and ES-F) increasing the number of excited state minima from 7 to $10 ; \mathbf{A}^{\prime}$ and $\mathbf{A}^{\prime \prime}$ conformers evolved towards the same minimum.

Although TICT formation has been largely studied, ${ }^{35-40}$ the concerted rotation around two dihedral angles has been less explored. Recently, G. Haberhauer ${ }^{41}$ has studied fluorophores where donor and acceptor groups have a predetermined sequence and has used, similar to our work, two independent rotations, originating from the presence of $\pi$-conjugated, $\mathrm{NH}$ linked moieties, to combine TICT and PLICT ${ }^{42}$ (or PICT, planarised intramolecular charge transfer states). ${ }^{18}$

In our case, the charge transfer is possible because, upon rotation around angle $\alpha_{1}$, the $\pi$ orbitals of acridone conjugated with the lone-pair of the nitrogen bridge give rise to a good electron donating group compared to a good cytosine acceptor. Simple rotation around $\alpha_{2}$, where the $\mathrm{NH}$ bridge remains within the cytosine plane, does not allow the formation of a TICT state and only transition states are detected. For a given $\alpha_{1}$, rotation around the $\alpha_{2}$ dihedral leads to the most favourable disposition of the two aromatic groups.

Coming to the spectroscopic aspects, our computations show that for all conformers, the first Franck-Condon excited state $S_{1}$ is well-separated in energy from the second state by at least $12 \mathrm{kcal} \mathrm{mol}^{-1}$. Moreover, the $\mathrm{S}_{0} \rightarrow \mathrm{S}_{1}$ transition energy is quite similar for all conformers (Table 3), their difference not exceeding $1.6 \mathrm{kcal} \mathrm{mol}^{-1}$. Therefore, the absorption band in Fig. 2 is in fact an envelope of the transitions associated with the four conformers. A closer comparison between the experimental absorption spectrum (Fig. 2) and the computed transition energies (Table 3) shows that the latter are overestimated. This inaccuracy is due to the fact that (i) the functional M062X, used in our computations, overestimates the electronic transition energies but without a drift ${ }^{43}$ between the locally excited and charge transfer transitions and (ii) vibrational/thermal effects are not taken into account. The contribution of each one conformer $i$ to the Franck-Condon excited state $p_{\mathrm{FC}}$ is given as $p_{\mathrm{GS} i} \times f_{i} / \sum\left(p_{\mathrm{GS} i} \times f_{i}\right)$, where $f$ is the oscillator strength (Table 3 ). The average computed oscillator strength $\langle f\rangle=\sum\left(p_{\mathrm{GS} i} \times f_{i}\right) / 100$ is 0.12 , a value in line with the maximum molar extinction coefficient of $5300 \mathrm{~mol}^{-1} \mathrm{~L} \mathrm{~cm}^{-1}$ determined experimentally (Fig. 2). Moreover, the computed Stokes shifts are $5-8 \mathrm{kcal} \mathrm{mol}^{-1}$, not very different from the experimental values, 7 and $11 \mathrm{kcal} \mathrm{mol}^{-1}$, determined by either considering the shoulder or the peak of the fluorescence spectrum (Fig. 2).

From steady-state and time-resolved experiments, we deduce that the emission spectrum is correlated mainly with a time constant of $7.9 \mathrm{~ns}$. A second time-constant of $0.5 \mathrm{~ns}$, whose amplitude is larger at shorter wavelengths, contributes by less than $4 \%$ to the emitted photons (Table 1 ). It could be correlated with the high energy shoulder (Fig. 2a), as suggested from experiments performed with GLAC in acetonitrile (Fig. $2 \mathrm{~b}$ and Table 1). Moreover, from the anisotropy values recorded on the 100 ps scale (Fig. 4), it appears that the emitting species with a lifetime of $0.5 \mathrm{~ns}$ is characterised by higher anisotropy. Given these considerations on the polarisation of the electronic transitions associated with the fluorescent species, we propose, at a first approach, a possible correlation between experimental and computed results. Thus, ES-A and ES-D, characterised by a fluorescence anisotropy of 0.21 and 0.17 , respectively (Table 5), are candidates for the 7.9 ns constant. Conversely, ES-B and ES-C for which the computed anisotropies are higher (0.40 and 0.35 , respectively) are candidates for the $0.5 \mathrm{~ns}$ time constant. One could think that, by some coincidence, the fluorescence dynamics are identical for the emitting minima of each pair. Yet, in view of the difference in the oscillator strength values calculated for each pair (Table 4: 0.20 and 0.10 for the first one, 0.11 and 0.15 for the second), together with the precision of our TCSPC measurements (error in the time constants: $0.1 \mathrm{~ns}$ ), such a scenario does not seem plausible. This is particularly true for the largest time constant.

The quenching of the fluorescence stemming from a given conformer $\mathbf{X}$ depends on its propensity to undergo $\mathbf{X} \rightarrow \mathbf{Y}$ isomerization toward another conformer $\mathbf{Y}$, emitting or not, which is determined by the energy barrier $\Delta E_{\mathbf{X Y}}$. These values, calculated considering as zero the excited state minimum ES-X, are shown in Table 6.

We also report the excess of energy, in respect to this barrier, resulting from the Franck-Condon transition, $\Delta E_{\mathrm{FC}}$. Although the loss of excess energy (vibrational relaxation, thermalisation) is expected to be much faster than the isomerisation process, we cannot rule out that, to some extent, it may favour overcoming the barrier. Finally, in Table 6 we report the half-life $t_{1 / 2}$ of $\mathbf{X}$, in respect to the transformation $\mathbf{X} \rightarrow \mathbf{Y}$, determined via the Eyring equation ${ }^{44}$ as follows:

$$
k=\kappa \cdot\left(k_{\mathrm{B}} T / h\right) \cdot \exp (-\Delta E / R T) \quad \text { and } t_{1 / 2}=\ln (2) / k
$$

where, $k_{\mathrm{B}}, h$ and $R$ are, respectively, the Boltzmann, Planck and gas constants and $T$ is the absolute temperature; the transmission coefficient $\kappa$ was set to 1 . The values are not expected to correspond to the experimentally determined lifetimes, but simply indicate differences in the dynamical behaviour of the various conformers. 
Table 6 Single step energy barriers that may affect transformation of isomer $\mathbf{X}$ to isomer $\mathbf{Y}$ (and vice versa) in the first excited state of $\mathrm{GLAC}^{-1}$. $\Delta \Delta G$ : energy barriers with respect to the $X$ and $Y$ minimum. $t_{1 / 2}$ : half times of $\mathbf{X}$ and $\mathbf{Y}$ determined via the Eyring equation. $\Delta E_{\mathrm{FC}}$ : excess of energy with respect to the barrier, resulting from the Franck-Condon excitation of $\mathbf{X}$ and $\mathrm{Y}$ calculated as: $\Delta E_{\mathrm{FC}}=\left(\Delta \Delta G_{\text {ground state }}+\right.$ energy transition -

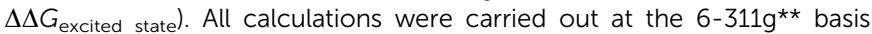
set level

\begin{tabular}{|c|c|c|c|c|c|c|}
\hline XY & $\begin{array}{l}\Delta \Delta G^{a} \\
\mathbf{X} \rightarrow \mathbf{Y}\end{array}$ & $\begin{array}{l}\Delta \Delta G^{a} \\
\mathbf{X} \leftarrow \mathbf{Y}\end{array}$ & $\begin{array}{l}t_{1 / 2}^{b} \\
\mathbf{X} \rightarrow \mathbf{Y}\end{array}$ & $\begin{array}{l}t_{1 / 2}^{b} \\
\mathbf{X} \leftarrow \mathbf{Y}\end{array}$ & $\begin{array}{l}\Delta E_{\mathrm{FC}}^{a} \\
\mathbf{X} \rightarrow \mathbf{Y}\end{array}$ & $\begin{array}{l}\Delta E_{\mathrm{FC}}{ }^{a} \\
\mathbf{X} \leftarrow \mathbf{Y}\end{array}$ \\
\hline AB & 5.2 & 4.6 & 681 & 210 & 5.3 & 9.3 \\
\hline $\mathbf{A B}^{*}$ & 5.1 & 4.5 & 576 & 210 & 5.3 & 9.3 \\
\hline $\mathbf{A} \mathbf{E}^{\prime}$ & 5.3 & 3.5 & 805 & 39 & 5.2 & - \\
\hline $\mathbf{A} \mathbf{F}^{\prime}$ & 5.4 & 3.3 & 952 & 28 & 5.1 & - \\
\hline $\mathbf{B E}^{\prime \prime}$ & 2.4 & 2.9 & 6 & 14 & 6.4 & - \\
\hline $\mathbf{B F}^{\prime \prime}$ & 2.3 & 3 & 5 & 17 & 6.5 & - \\
\hline $\mathbf{C}^{\prime} \mathbf{D}^{\prime \prime}$ & 2.3 & 3 & 5 & 17 & 6.3 & 6.8 \\
\hline $\mathbf{C}^{\prime} \mathbf{F}^{\prime}$ & 3.9 & 3.1 & 77 & 20 & 4.6 & - \\
\hline $\mathbf{C}^{\prime \prime} \mathbf{D}^{\prime}$ & 2.8 & 2.9 & 12 & 14 & 6.5 & 6.6 \\
\hline $\mathbf{C}^{\prime \prime} \mathbf{E}^{\prime}$ & 3.9 & 2.9 & 77 & 14 & 5.7 & - \\
\hline $\mathbf{D}^{\prime} \mathbf{F}^{\prime \prime}$ & 3.3 & 4.1 & 28 & 108 & 6.2 & - \\
\hline $\mathbf{D}^{\prime \prime} \mathbf{E}^{\prime \prime}$ & 2.8 & 3.3 & 12 & 28 & 7.0 & - \\
\hline
\end{tabular}

${ }^{a}$ In kcal mol ${ }^{-1} \cdot{ }^{b}$ In ps.

From Table 6, we could remark on two extreme cases. On the one side, the highest energy barriers, the lowest excess energy $\Delta E_{\mathrm{FC}}$ and the longest $t_{1 / 2}$ values are encountered for conformer A. Therefore, we deduce that this conformer is responsible for the fluorescence component characterised by a lifetime of $7.9 \mathrm{~ns}$. On the other side, conformer $\mathbf{B}$ is characterised by the lowest energy barriers and the highest excess energy, making its evolution toward TICT states highly probable. Such dynamical factors, associated with the fact that the contribution of $\mathbf{B}$ to the Franck-Condon population is only $2 \%$, preclude detection of fluorescence arising from B. Therefore, we assign the $0.5 \mathrm{~ns}$ fluorescence lifetime to $\mathbf{C}$ conformers, characterised by intermediate $t_{1 / 2}$ values and energy barriers, their evolution toward TICT states (ES-E and ES-F) being only partial. TICT states are known to be stabilised in polar solvents. ${ }^{45}$ Accordingly, one could assign to this effect the observed decrease of fluorescence quantum yield of GLAC in water compared to acetonitrile (0.09 vs. 0.22). Moreover, as the energy barriers leading to TICT are lower for ES-C than for ES-A conformers, the fluorescence lifetime of the former undergoes more important decrease (from 1.3 to $0.5 \mathrm{~ns}$ ) than that of the latter (from 9.6 to $7.9 \mathrm{~ns}$ ). However, in the present case, such an interpretation is oversimplified because GLAC in neutral aqueous solution is present as a monoanion. An additional interpretation could be based on the behaviour of the ground state. In the case of the nonemitting ES-E and ES-F conformers, the energy gap between $S_{0}$ and $S_{1}$ is very low, possibly arising from higher destabilisation of the corresponding ground-state twisted structures, due to the non-equilibrium solvation. We expect that this destabilization is higher in aqueous buffer than in acetonitrile.

Finally, regarding our experimental observations on the ps time-scale, we stress that the fluorescence upconversion technique is capable of probing photons associated with quantum yields as low as $10^{-6} \cdot{ }^{46}$ In other words, it can detect emitting species whose fingerprint is not present in the steady-state fluorescence spectra. In a qualitative way, we attribute the important decay at $430 \mathrm{~nm}$ observed for $\mathrm{GLAC}^{-1}$ aqueous solutions to transformations of the $\mathbf{C}$ conformers and the weak rise at $515 \mathrm{~nm}$ to the appearance of the $\mathbf{D}$ conformer, which subsequently evolves toward TICT states. We already mentioned $\mathbf{C}$ as a candidate for the $0.5 \mathrm{~ns}$ time constant. However, as the values reported in Table 6 for the mirror images $\mathbf{C}^{\prime}$ and $\mathbf{C}^{\prime \prime}$ are somewhat different, it is possible that their dynamics are also different. Alternatively, the decays at short time-scales could have a nonexponential behaviour and our fits provide only a phenomenological picture. A temperature dependent study of the ultrafast decay and rise should certainly bring valuable information about these processes. But such measurements are very delicate on this time-scale and our setup is not equipped to this end. In addition, at this time-scale, we also have contribution from the solvent relaxation. We assign to this process the 1 ps time-constant, which is typical for aqueous solutions. ${ }^{47,48}$

\section{Conclusion and outcome}

We have presented a study on the fluorescence of GLAC, a recently described potent inhibitor of glycogen phosphorylase. The most important part of our work dealt with free GLAC in aqueous neutral solution, where it exists in its mono-anionic form. Combination of multiscale time-resolved spectroscopy with quantum chemistry calculations revealed a quite complex behaviour in the excited state relaxation.

Our calculations evidenced the existence of four classes of conformers in the ground state of $\mathrm{GLAC}^{-1}$, resulting from mutual rotation of the acridone and cytosine components around two dihedral angles. The number of conformers further increases in the first electronically excited state where the formation of twisted intermolecular charge transfer states results in partial quenching of the fluorescence. The fingerprints of several conformers were detected experimentally in the fluorescence dynamics; they were identified using the polarisation of their electronic transitions as criterium. Despite this diversity, the steady-state fluorescence spectrum, characterised by a quantum yield of 0.09 , arises from a single conformer whose lifetime is $7.9 \mathrm{~ns}$.

GLAC is the first fluorescent inhibitor of glycogen phosphorylase. In neutral aqueous solutions, its fluorescence peaks around $465 \pm 10 \mathrm{~nm}$, both in its free form and when bound to the enzyme. Though its fluorescence decreases upon binding, it is still possible to properly study its dynamic behaviour. ${ }^{49}$ The observation that, on the one hand, GLAC appears to adopt a bisanionic form within its complex with $\mathrm{GP}^{4}$ and, on the other, free $\mathrm{GLAC}^{-2}$ in solution is not emitting (Fig. 3), suggests the existence of a subtle interplay between rotation and deprotonation processes. In order to fully assess these processes, computations taking into account the detailed local environment within the enzyme pocket are currently in progress. The identification of the factors responsible for the partial quenching of GLAC fluorescence will allow the design of novel fluorescent 1-aryl- $\beta$-D-glucopyranose derivatives.

Beyond the importance of the present study related to the potential therapeutic action of 1 -aryl- $\beta$-D-glucopyranose derivatives 
and their use as labels for probing dynamical interactions with glycogen phosphorylase, our computational work may be viewed as a more general showcase. As a matter of fact, although numerous computational studies describe excited state surfaces involving mutual rotation between two aromatic moieties, here we show how two independent rotations may rule the process in a subtle equilibrium, potentially leading to the formation of a TICT state. Rotation of the dihedral angle $\alpha_{2}$ is responsible for charge transfer which is possible only if the nitrogen lone pair of the bridge is part of the $\pi$ orbitals of the acridone moiety. In other words, only in this case, the acridone moiety behaves as a donor with respect to the cytosine moiety that acts like an acceptor. Rotation around $\alpha_{2}$ simply assists $\alpha_{1}$ for finding the most favourable disposition of the two aromatic groups. Simple rotation around $\alpha_{2}$, where the $\mathrm{NH}$ bridge remains planar with cytosine, does not allow the formation of a TICT state and transition states are detected.

\section{Conflicts of interest}

There are no conflicts to declare.

\section{Acknowledgements}

This work was supported by Heracleitus II (awarded to M. M), LASERLAB-EUROPE (FP7 No. 284464) and "Progetto Bandiera", N-CHEM (to A. V). K. M. acknowledges funding from the Hellenic National Scholarships Foundation through a "Strengthening of Human Resources through Doctoral Research" program, co-financed by the European Union (European Social Fund ESF) and Greek national funds through the Operational Program "Human Resource Development, Education and Lifelong Learning”' 2014-2020.

\section{References}

1 E. D. Chrysina, Mini-Rev. Med. Chem., 2010, 10, 1093-1101.

2 T. Gimisis, Mini-Rev. Med. Chem., 2010, 10, 1127-1138.

3 L. Agius, Mol. Aspects Med., 2015, 46, 34-45.

4 M. Mamais, A. Degli Esposti, V. Kouloumoundra, T. Gustavsson, F. Monti, A. Venturini, E. D. Chrysina, D. Markovitsi and T. Gimisis, Chem. - Eur. J., 2017, 23, 8800-8805.

5 T. Hadjiloi, C. Tiraidis, E. D. Chrysina, D. D. Leonidas, N. G. Oikonomakos, P. Tsipos and T. Gimisis, Bioorg. Med. Chem., 2006, 14, 3872-3882.

6 C. Tiraidis, K.-M. M. Alexacou, S. E. Zographos, D. D. Leonidas, T. Gimisis and N. G. Oikonomakos, Protein Sci., 2007, 16, 1773-1782.

7 Y. Liu, C. H. Wolstenholme, G. C. Carter, H. Liu, H. Hu, L. S. Grainger, K. Miao, M. Fares, C. A. Hoelzel, H. P. Yennawar, G. Ning, M. Du, L. Bai, X. Li and X. Zhang, J. Am. Chem. Soc., 2018, 8-11.

8 O. Tietz, J. Kaur, A. Bhardwaj and F. R. Wuest, Org. Biomol. Chem., 2016, 14, 7250-7257.

9 G. Leriche, A. C. Chen, S. Kim, D. J. Selkoe and J. Yang, ACS Chem. Neurosci., 2016, 7, 40-45.
10 L. C. Kenmogne, R. Maltais and D. Poirier, Bioorg. Med. Chem. Lett., 2016, 26, 2179-2183.

11 A. Thorarensen, R. W. Sarver, F. Tian, A. Ho, D. L. Romero and K. R. Marotti, Bioorg. Med. Chem. Lett., 2007, 17, 4646-4649.

12 M. Kondo, I. A. Heisler and S. R. Meech, J. Phys. Chem. B, 2010, 114, 12859-12865.

13 L. Dehmel, F. Berndt, M. Weinberger, M. Sajadi, I. Ioffe, H.-A. Wagenknecht and N. P. Ernsting, Phys. Chem. Chem. Phys., 2016, 18, 6813-6820.

14 J. S. Beckwith, A. Rosspeintner, G. Licari, M. Lunzer, B. Holzer, J. Fröhlich and E. Vauthey, J. Phys. Chem. Lett., 2017, 8, 5878-5883.

15 D. Zhong, S. K. Pal, C. Wan and A. H. Zewail, Proc. Natl. Acad. Sci. U. S. A., 2001, 98, 11873-11878.

16 C. Martín, M. Gil, B. Cohen and A. Douhal, Langmuir, 2012, 28, 6746-6759.

17 I. Vayá, P. Bonancía, M. C. Jiménez, D. Markovitsi, T. Gustavsson and M. A. Miranda, Phys. Chem. Chem. Phys., 2013, 15, 4727.

18 P. H. Gore and G. K. Hughes, J. Chem. Soc., 1959, 1615-1616. 19 M. M. Barnett, A. H. C. P. Gillieson and W. O. Kermack, J. Chem. Soc., 1934, 433-435.

20 M. Mamais, V. Kouloumoundra, E. Smyrli, P. Grammatopoulos, E. D. Chrysina and T. Gimisis, Tetrahedron Lett., 2015, 56, 5549-5552.

21 T. Gustavsson, L. Cassara, V. Gulbinas, G. Gurzadyan, J.-C. Mialocq, S. Pommeret, M. Sorgius and P. van der Meulen, J. Phys. Chem. A, 1998, 102, 4229-4245.

22 M. Caricato, B. Mennucci, J. Tomasi, F. Ingrosso, R. Cammi, S. Corni and G. Scalmani, J. Chem. Phys., 2006, 124, 94107.

23 J. Tomasi, B. Mennucci and R. Cammi, Chem. Rev., 2005, 105, 2999-3093.

24 M. J. Frisch, G. W. Trucks, H. B. Schlegel, G. E. Scuseria, M. A. Robb, J. R. Cheeseman, G. Scalmani, V. Barone, G. A. Petersson, H. Nakatsuji, X. Li, M. Caricato, A. V. Marenich, J. Bloino, B. G. Janesko, R. Gomperts, B. Mennucci, H. P. Hratchian, J. V. Ortiz, A. F. Izmaylov, J. L. Sonnenberg, D. Williams-Young, F. Ding, F. Lipparini, F. Egidi, J. Goings, B. Peng, A. Petrone, T. Henderson, D. Ranasinghe, V. G. Zakrzewski, J. Gao, N. Rega, G. Zheng, W. Liang, M. Hada, M. Ehara, K. Toyota, R. Fukuda, J. Hasegawa, M. Ishida, T. Nakajima, Y. Honda, O. Kitao, H. Nakai, T. Vreven, K. Throssell, J. A. Montgomery Jr., J. E. Peralta, F. Ogliaro, M. J. Bearpark, J. J. Heyd, E. N. Brothers, K. N. Kudin, V. N. Staroverov, T. A. Keith, R. Kobayashi, J. Normand, K. Raghavachari, A. P. Rendell, J. C. Burant, S. S. Iyengar, J. Tomasi, M. Cossi, J. M. Millam, M. Klene, C. Adamo, R. Cammi, J. W. Ochterski, R. L. Martin, K. Morokuma, O. Farkas, J. B. Foresman and D. J. Fox, Gaussian 16, Revision B.01, Gaussian Inc., Wallingford CT, 2016.

25 Y. Zhao and D. G. Truhlar, Acc. Chem. Res., 2008, 41, 157-167.

26 R. Improta, Phys. Chem. Chem. Phys., 2008, 10, 2656-2664.

27 F. Santoro, V. Barone and R. Improta, J. Am. Chem. Soc., 2009, 131, 15232-15245. 
28 R. Improta, G. Scalmani, M. J. Frisch and V. Barone, J. Chem. Phys., 2007, 127, 74504.

29 R. Improta, V. Barone, G. Scalmani and M. J. Frisch, J. Chem. Phys., 2006, 125, 54103.

30 C. Adamo, T. Le Bahers, M. Savarese, L. Wilbraham, G. García, R. Fukuda, M. Ehara, N. Rega and I. Ciofini, Coord. Chem. Rev., 2015, 304-305, 166-178.

31 A. M. Brouwer, Pure Appl. Chem., 2011, 83, 2213-2228.

32 T. Yanai, D. P. Tew and N. C. Handy, Chem. Phys. Lett., 2004, 393, 51-57.

33 M. Siegmund and J. Bendig, Z. Naturforsch., A: Phys. Sci., 1980, 35, 1076-1086.

34 L. Martínez-Fernández, A. J. Pepino, J. Segarra-Martí, J. Jovaišaitè, I. Vaya, A. Nenov, D. Markovitsi, T. Gustavsson, A. Banyasz, M. Garavelli and R. Improta, J. Am. Chem. Soc., 2017, 139, 7780-7791.

35 D. Su, C. L. Teoh, L. Wang, X. Liu and Y.-T. Chang, Chem. Soc. Rev., 2017, 46, 4833-4844.

36 F. Monti, A. Venturini, A. Nenov, F. Tancini, A. D. Finke, F. Diederich and N. Armaroli, J. Phys. Chem. A, 2015, 119, 10677-10683.

37 Conformational Analysis of Molecules in Excited States, ed. J. Waluk, Wiley-VCH, 2000.
38 Z. R. Grabowski, K. Rotkiewicz and W. Rettig, Chem. Rev., 2003, 103, 3899-4031.

39 P. B. Coto, L. Serrano-Andrés, T. Gustavsson, T. Fujiwara and E. C. Lim, Phys. Chem. Chem. Phys., 2011, 13, 15182-15188.

40 D. Markovitsi, H. Sigal, C. Ecoffet, P. Millié, F. Charra, C. Fiorini, J.-M. Nunzi, H. Strzelecka, M. Veber and C. Jallabert, Chem. Phys., 1994, 182, 69-80.

41 G. Haberhauer, Chem. - Eur. J., 2017, 23, 9288-9296.

42 G. Haberhauer, R. Gleiter and C. Burkhart, Chem. - Eur. J., 2016, 22, 971-978.

43 C. Adamo and D. Jacquemin, Chem. Soc. Rev., 2013, 42, 845-856.

44 H. Eyring, Chem. Rev., 1935, 17, 65-77.

45 Z. R. Grabowski, K. Rotkiewicz and W. Rettig, Chem. Rev., 2003, 103, 3899-4032.

46 P. Changenet-Barret, T. Gustavsson, D. Markovitsi and I. Manet, ChemPhysChem, 2016, 17, 1264-1272.

47 R. Jimenez, G. R. Fleming, P. V. Kumar and M. Maroncelli, Nature, 1994, 369, 471-473.

48 W. Jarzeba, G. C. Walker, A. E. Johnson, M. A. Kahlow and P. F. Barbara, J. Phys. Chem., 1988, 92, 7039-7041.

49 E. D. Chrysina, T. Gimisis, T. Gustavsson, M. Mamais and D. Markovitsi, Unpubl. results. 